\title{
Long-distance dissipation-assisted transport of entangled states via a chiral waveguide
}

\author{
Wai-Keong Mok $\odot,{ }^{1,2, *}$ Davit Aghamalyan, ${ }^{2}$ Jia-Bin You, ${ }^{1}$ Tobias Haug $\odot,{ }^{2}$ Wenzu Zhang $\odot,{ }^{1}$ \\ Ching Eng Png, ${ }^{1}$ and Leong-Chuan Kwek ${ }^{2,3,4}$ \\ ${ }^{1}$ Department of Electronics and Photonics, Institute of High Performance Computing, \\ 1 Fusionopolis Way, 16-16 Connexis, 138632 Singapore \\ ${ }^{2}$ Centre for Quantum Technologies, National University of Singapore, 3 Science Drive 2, 117543 Singapore \\ ${ }^{3}$ MajuLab, CNRS-UNS-NUS-NTU International Joint Research Unit, UMI No. 3654, 117543 Singapore \\ ${ }^{4}$ National Institute of Education and Institute of Advanced Studies, Nanyang Technological University, \\ 1 Nanyang Walk, 637616 Singapore
}

(Received 8 November 2019; accepted 9 March 2020; published 26 March 2020)

\begin{abstract}
Quantum networks provide a prominent platform for realizing quantum information processing and quantum communication, with entanglement being a key resource in such applications. Here we describe the dissipative transport protocol for entangled states, where entanglement stored in the first node of a quantum network can be transported with high fidelity to the second node via a one-dimensional chiral waveguide. In particular, we exploit the directional asymmetry in chirally coupled single-mode ring resonators to transport entangled states. For the fully chiral waveguide, Bell states, multipartite $W$ states, and and Dicke states can be transported with fidelity as high as 0.954 , despite the fact that the communication channel is noisy. Our proposal can be utilized for the long-distance distribution of multipartite entangled states between the quantum nodes of an open quantum network.
\end{abstract}

DOI: 10.1103/PhysRevResearch.2.013369

\section{INTRODUCTION}

Quantum networks [1,2] are essential for realizing distributed quantum computing and large-scale quantum communication, with entanglement being a key resource in such applications. In this context, the main task and at the same time an outstanding challenge is the high-fidelity transfer of quantum states over long distances despite having noise and dissipation present in the quantum channel [3]. It is well known that in open quantum systems, dissipation arises as a result of the system coupling with the reservoir [4], which consequently causes decoherence in the system. In this paper we show that the dissipative channel can be used for transporting entangled states. In general, long-distance processes in open quantum systems are challenging because of non-Markovian effects due to the non-negligible time delay between the nodes of a quantum network. Non-Markovianity has been shown to be detrimental to both quantum state transfer and entanglement generation between the nodes [5,6].

There have been several theoretical proposals [7-17] as well as actual experimental realizations [18-20] for the quantum state transfer (QST) of a single qubit in quantum optical networks, where fast information transfer is achieved with the help of photons (flying qubits). In all the above proposals,

\footnotetext{
*waikeong_mok@u.nus.edu

Published by the American Physical Society under the terms of the Creative Commons Attribution 4.0 International license. Further distribution of this work must maintain attribution to the author(s) and the published article's title, journal citation, and DOI.
}

there are a few demanding requirements that are hard to meet experimentally: external control pulses that have nontrivial temporal shapes (photonic wave packets are required to be time symmetric) and time-dependent cavity-atom and fiberatom interaction strengths. Moreover, there are no existing protocols for long-distance entanglement transfer in the optical frequency domain.

On the other hand, spin chains can alleviate the issue of sensitive control of system parameters and realize quantum systems with minimal control (coupling constants are fixed in time) and entanglement transfer has been demonstrated in several theoretical papers [21-28] in Heisenberg-type spin chains. However, these systems can only realize shortdistance state transfer, as experimentally one is limited by the number of spins. It is also widely believed that increasing the length of a spin chain will worsen transfer fidelities due to dispersion effects [28].

Quite remarkably, using chiral waveguides, the merits of quantum optical networks (fast information transfer with flying qubits) and spin chain networks (minimal control over system parameters) can be combined. In quantum optics, chirality arises, for instance, in atom-waveguide coupled systems when the symmetry of photon emission in the left and right directions is broken [29]. This effect appears as a result of spin-orbit coupling and has been experimentally demonstrated in photonic waveguides [30]. Chiral systems have been shown to be fruitful for realizing quantum networks [14,31,32]. In Ref. [33] it was argued that the maximum achievable concurrence between two atoms is 1.5 times higher as compared to the nonchiral counterparts.

Interestingly, systems with perfect chirality realize the paradigm of cascaded systems [34-36], where two systems 
are coupled unidirectionally without information backflow. Cascaded systems, even when separated by long distances, can then be described under the Born-Markov approximation with retardation effects accounted for by a simple redefinition of the time and phase of the second node [36] such that the resulting Markovian master equation does not contain source retardation. Physically, the time delay between the two nodes is not important for cascaded systems since there is no backaction from the target to the source, by definition. Here we exploit the Markovianity provided by cascaded systems as a suitable platform to achieve high-fidelity entanglement transfer, despite the noise being present in the quantum channel.

Motivated by Refs. [9,33], we couple ring cavities with chiral waveguides to obtain unidirectional effective coupling between the cavities. In particular, we benefit from Markovian dynamics due to the cascaded systems naturally arising from the high chirality. Each node of our quantum network consists of an $N$-particle atomic ensemble which is coupled to the ring cavity. It is important to highlight that ring cavities introduce greater control over the system compared to the bare-atomfiber coupled case, where the transport fidelity is significantly lowered due to all-to-all long-range interactions between the atoms. By suitably optimizing over the system parameters, we demonstrate the transport of maximally entangled Bell states, Dicke states, and $W$ states for up to 20 qubits. For clarity, we remark that the entanglement transport here is not necessarily QST, although the transport of $W$ states and Bell states can be applied to the QST of unknown qubit and qutrit states, respectively.

Compared to other schemes, our minimal control proposal has various advantages. First, the scheme works in the weakcoupling regime with no external driving field required. Also, the optimal transport of entanglement occurs dynamically. This potentially can lead to faster transport compared to steady-state schemes [31]. Moreover, the entanglement transport is not dependent on the distance between the atoms.

\section{CHIRAL WAVEGUIDE QED SYSTEM}

The system in consideration comprises two nodes coupled to a one-dimensional (1D) waveguide, shown in Fig. 1(a). Each node comprises $N$ qubits coupled to a single cavity mode where the transition frequencies of the qubits and resonant frequency of the cavity are $\omega_{l}^{(j)}$ and $\omega_{c j}$, respectively. The atom-cavity coupling strength is given by $g_{l}^{(j)}$. The bosonic operators for the cavity mode are $a_{j}^{\dagger}$ and $a_{j}$, satisfying the canonical commutation relation $\left[a_{j}, a_{j^{\prime}}^{\dagger}\right]=\delta_{j j^{\prime}}$. The waveguide is treated as a common reservoir, with bosonic operators $b_{\lambda}^{\dagger}(\omega)$ and $b_{\lambda}(\omega)$ satisfying the commutation relation $\left[b_{\lambda}(\omega), b_{\lambda^{\prime}}^{\dagger}\left(\omega^{\prime}\right)\right]=\delta_{\lambda \lambda^{\prime}} \delta\left(\omega-\omega^{\prime}\right)$. The interaction strength between the cavities and the waveguide (at position $x_{j}$ ) is characterized by the decay rate $\gamma_{\lambda}$. Here we assume that cavity losses into nonwaveguide modes are negligible, which can be realized in photonic waveguides with $\beta$ factors close to unity [37]. The spontaneous decay of the qubits is described by an interaction with independent baths at a decay rate $\Gamma_{j l}$, where the first index denotes the cavity and the second index denotes
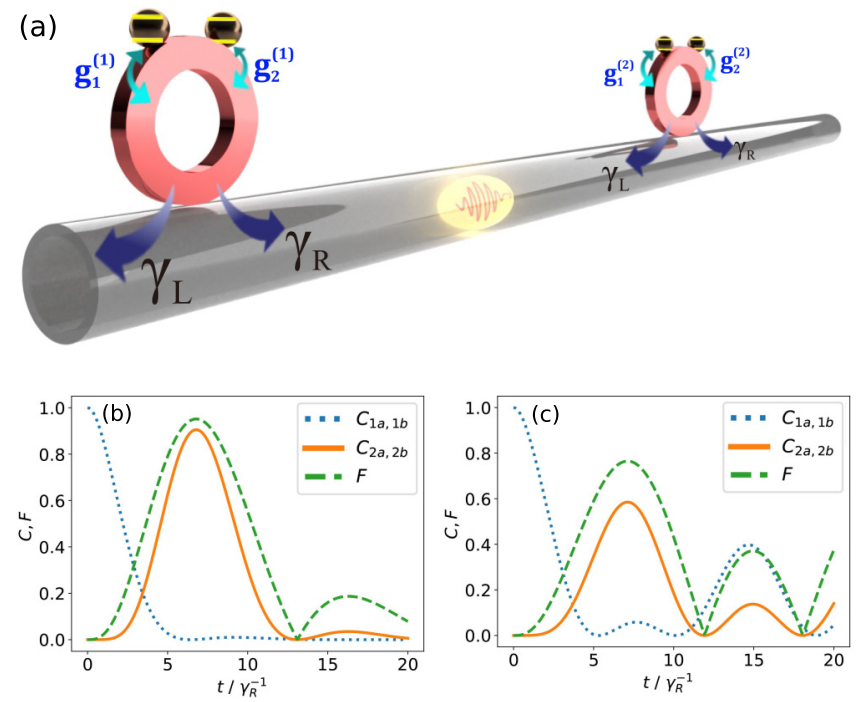

FIG. 1. (a) Proposed setup for entanglement transport. Each node comprises $N$ qubits ( $N=2$ in the figure) coupled to a single cavity mode. Chirality is enforced by setting $\gamma_{L} \neq \gamma_{R}$. Concurrence and fidelity are shown for the transport of the Bell state $\left|\Psi^{+}\right\rangle$, with $C_{1 a, 1 b}$ and $C_{2 a, 2 b}$ denoting the qubit concurrence in the left and right nodes, respectively. (b) Chiral coupling with $\gamma_{L}=0$. (c) Nonchiral coupling with $\gamma_{L}=\gamma_{R}$ and $k D=\pi$. Cavity-atom coupling is set at the optimal value $g_{1}=g_{2}=0.3 \gamma_{R}$.

the qubit. The bath operators $c_{l}^{(j) \dagger}(\omega)$ and $c_{l}^{(j)}(\omega)$ satisfy the commutation relation $\left[c_{l}^{(j)}(\omega), c_{l^{\prime}}^{\left(j^{\prime}\right) \dagger}\left(\omega^{\prime}\right)\right]=\delta_{j j^{\prime}} \delta_{l l^{\prime}} \delta\left(\omega-\omega^{\prime}\right)$.

By tracing out the waveguide mode and applying the BornMarkov approximation, the Lindblad master equation for the system can be found as [31] (setting $\hbar=1$, details in the Appendix)

$$
\begin{aligned}
\dot{\rho}= & -i\left[H_{\mathrm{eff}}, \rho\right]+\gamma_{L} \mathcal{D}\left[e^{i k x_{1}} a_{1}+e^{i k x_{2}} a_{2}\right] \rho \\
& +\gamma_{R} \mathcal{D}\left[e^{-i k x_{1}} a_{1}+e^{-i k x_{2}} a_{2}\right] \rho+\sum_{j, l} \Gamma_{j l} \mathcal{D}\left[\sigma_{l}^{(j)}\right] \rho,
\end{aligned}
$$

with the effective Hamiltonian

$$
\begin{aligned}
H_{\mathrm{eff}}= & \sum_{j, l}\left[\omega_{l}^{(j)} \sigma_{l}^{(j) \dagger} \sigma_{l}^{(j)}+\omega_{c j} a_{j}^{\dagger} a_{j}+g_{l}^{(j)}\left(a_{j}^{\dagger} \sigma_{l}^{(j)}+\text { H.c. }\right)\right] \\
& -i \frac{\gamma_{L}}{2}\left(e^{i k D} a_{1}^{\dagger} a_{2}-\text { H.c. }\right)-i \frac{\gamma_{R}}{2}\left(e^{i k D} a_{2}^{\dagger} a_{1}-\text { H.c. }\right), \quad(2)
\end{aligned}
$$

where $D=\left|x_{2}-x_{1}\right|$ is the distance between the nodes. The Lindblad superoperator in the master equation is given by $\mathcal{D}[\hat{\mathcal{O}}] \rho=\mathcal{O} \rho \mathcal{O}^{\dagger}-\frac{1}{2}\left\{\mathcal{O}^{\dagger} \mathcal{O}, \rho\right\}$. In the following, we will study the transport of entangled qubit states between the nodes mediated by the waveguide. The case of $N=2$ is first presented to illustrate Bell state transport.

\section{TRANSPORT OF BELL STATES WITH CHIRAL COUPLINGS}

Here we exploit the directional asymmetry by using a chiral light-matter interface, with $\gamma_{L}=0$ and $\gamma_{R} \neq 0$ [9]. Here chirality is defined as $\chi \equiv\left(\gamma_{R}-\gamma_{L}\right) /\left(\gamma_{R}+\gamma_{L}\right)$, with the perfectly chiral case corresponding to $\chi=1$. Using chiral couplings, the setup is essentially a cascaded quantum system 
[34] where the first node is coupled to the second node unidirectionally without backflow of information. In this case, the setup we consider can be used to study long-distance entanglement transport despite the Born-Markov approximation used, since retardation effects in a cascaded quantum system are accounted for by a simple redefinition of the time and phase of the second node [36].

For simplicity, we assume that the qubit decay rates are much smaller than the cavity decay rates and can be neglected, and the nodes are identical, i.e., $\omega_{l}^{(j)}=\omega_{0}, \omega_{c j}=\omega_{c}$, and $g_{l}^{(j)}=g_{j}$ for all $j, l \in\{1,2\}$. The qubits in the first node are denoted by $1 a, 1 b$ while the qubits in the second node are denoted by $2 a, 2 b$. We first prepare the qubits $1 a, 1 b$ in the Bell state $\left|\Psi^{+}\right\rangle=\frac{1}{\sqrt{2}}(|e g\rangle+|g e\rangle)$ and consider resonant conditions $\omega_{c}=\omega_{0}$ with cavity coupling strength $g_{1}=g_{2}=$ $0.3 \gamma_{R}$. For the case of $N=2$, the entanglement of the twoqubit mixed state $\rho$ is measured by the concurrence, which is defined as

$$
C=\max \left(0, \lambda_{1}-\lambda_{2}-\lambda_{3}-\lambda_{4}\right),
$$

where $\lambda_{i}, i=1,2,3,4$, are the eigenvalues of the matrix $\sqrt{\sqrt{\rho}} \tilde{\rho} \sqrt{\rho}$ in decreasing order, with $\tilde{\rho}=\left(\sigma_{y} \otimes \sigma_{y}\right) \rho^{*}\left(\sigma_{y} \otimes\right.$ $\sigma_{y}$ ) the spin-flipped state [38].

As shown in Fig. 1(b), the concurrence of $1 a, 1 b$ decreases to nearly zero at some time, while concurrence of $2 a, 2 b$ increases from zero to a maximum of around 0.91. The state fidelity of $2 a, 2 b$ compared to the initial Bell state is around 0.954 . This shows that good entanglement transport can be accomplished. For the case of perfect chirality $(\chi=1)$, due to the cascaded nature of the system, this result is independent of the distance $D$ between the qubits. For the nonchiral case in Fig. 1(c) where $\gamma_{L}=\gamma_{R}$, the maximum concurrence is only around 0.58 , even with the optimal distance of $k D=\pi$, where $k$ is the wave number of the photon $k=2 \pi / \lambda$, with $\lambda$ the corresponding wavelength. Comparing the fidelity of the qubit state of $2 a, 2 b$ [denoted by $\rho_{2}(t)$ ] with the initial entangled state of $1 a, 1 b$ [denoted by $\rho_{1}(0)$ ] such that $\mathcal{F}=$ $\left[\operatorname{Tr} \sqrt{\sqrt{\rho_{1}(0)} \rho_{2}(t) \sqrt{\rho_{1}(0)}}\right]^{2}$, Fig. 1(b) shows that the maximum fidelity transported $\mathcal{F}_{\max }$ is around 0.951 (green dashed line), a significant improvement over the nonchiral case in Fig. 1(c), which gives $\mathcal{F}_{\max } \approx 0.78$. Thus, chiral coupling drastically improves the entanglement transport between the nodes.

To find the optimal coupling $g_{1}=g_{2}=g$, we plot the maximum transported concurrence $C_{\max }$ of $2 a, 2 b$ against $g_{1}$ and $g_{2}$. As shown in Fig. 2(a), the transported concurrence is maximal $\left(C_{\max } \approx 0.905\right)$ around $g_{1}=g_{2} \approx 0.3 \gamma_{R}$. Intuitively, for small couplings, the entanglement does not transport effectively to the cavity, and thus the transport is weak. For strong couplings however, the Rabi oscillations between the cavity and the qubits become more significant, which is detrimental to the transport of entanglement via the waveguide. It can also be seen from Fig. 2(a) that $g_{1}=g_{2}$ is an optimal condition for good entanglement transport.

To illustrate the effect of chirality on the transport, we compare the maximum transported concurrence for different chirality. We comment that although non-Markovian effects should in general be taken into account if one considers long distances with imperfect chirality, this is not required
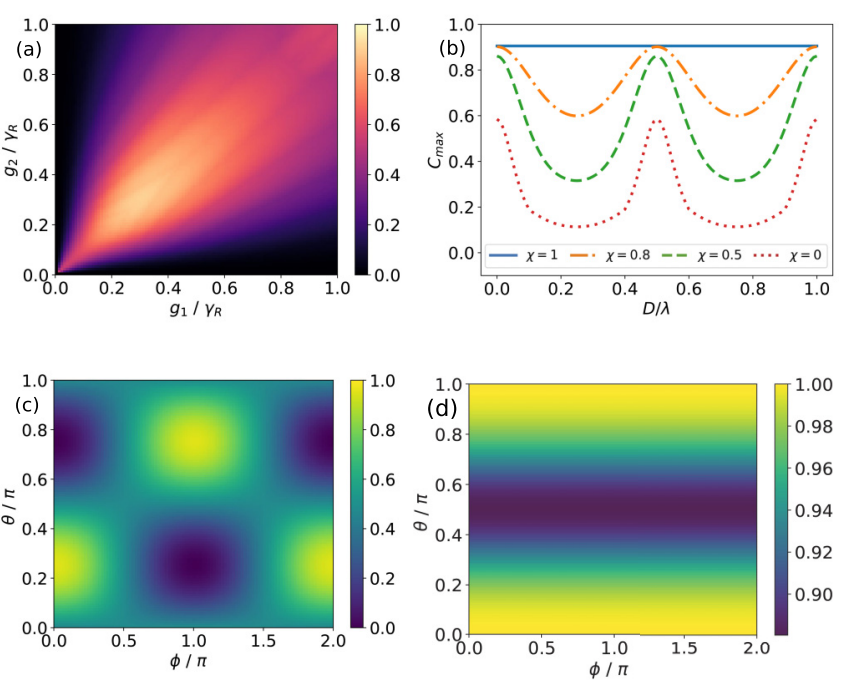

FIG. 2. (a) Maximum concurrence of $2 a, 2 b$ against $g_{1}, g_{2}$ showing the optimal point $g_{1}=g_{2}=0.3 \gamma_{R}$. (b) Maximum concurrence of $2 a, 2 b$ against internodal distance $D$. (c) Maximum fidelity of $2 a, 2 b$ against various initial states $\left|\psi_{\alpha}\right\rangle$. (d) Maximum fidelity of $2 a, 2 b$ against various initial states $\left|\psi_{\beta}\right\rangle$. The other parameters are $g_{1}=g_{2}=0.3 \gamma_{R}$, and $\Gamma_{j l}=0$.

as long as the entanglement is transported much faster than the timescale for information backflow to occur. For example, if the distance between the nodes is such that the time delay $\tau \gg \gamma_{R}^{-1}$, then the non-Markovian effects do not appear at the much shorter system timescale. As a result, the decay rate $\gamma_{L} \neq 0$ can be simply regarded as additional leakage of excitation from the second node. Our results indicate that such conditions can be easily achieved for sufficiently long waveguides. Figure 2(b) shows the comparison for different chirality. For the fully chiral waveguide $(\chi=1), C_{\max }$ is independent of the internodal distance $D$, as previously mentioned. This is simply due to the cascaded nature of the setup. However, when $\gamma_{L} \neq 0, C_{\max }$ depends on the distance between the nodes. The peak at $D=0.5 \lambda$ is a result of the spatial localization of the photon wave function between the nodes [5], resulting in less detrimental scattering effects, which contributes to excitation leakage. The sensitivity of $C_{\max }$ to fluctuations around this optimal point decreases as $\chi$ gets closer to 1 . In general, the entanglement transport worsens with decreasing chirality. Intuitively, this can be due to two factors: (i) leakage of excitation from the first node through the left port via $\gamma_{L}$, which decreases the probability of the second node being excited, and (ii) information backflow from the second node back to the first node, which can be detrimental to the transport process. Thus, using chirality, both problems can be addressed simultaneously, leading to good entanglement transport.

Next we look at the maximum transported fidelity with different initial states of $1 a, 1 b$. To this end, we prepare the qubits in system 1 in the state

$$
\left|\psi_{\alpha}\right\rangle=\cos \theta|e g\rangle+e^{i \phi} \sin \theta|g e\rangle, \quad \theta \in[0, \pi], \phi \in[0,2 \pi],
$$


while the qubits in system 2 are initially in the ground state. The cavities are all in the vacuum state initially. In Fig. 2(c), the maximum transported fidelity $\left(\mathcal{F}_{\max }=0.951\right)$ occurs near $(\phi=0, \theta=\pi / 4)$, which corresponds to the Bell state $\left|\Psi^{+}\right\rangle$. The case of $\mathcal{F}_{\max }=0$ occurs near $(\phi=\pi, \theta=\pi / 4)$, which corresponds to the Bell state $\left|\Psi^{-}\right\rangle$. This is because $\left|\Psi^{-}\right\rangle$is a dark state and thus does not decay with time. However, $\left|\Psi^{-}\right\rangle$ can be easily transported with the same fidelity of $\mathcal{F}_{\max }=$ 0.951 by imposing a $\pi$ phase difference between the two cavity-qubit couplings in the same node, i.e., $g_{1}^{(j)}=-g_{2}^{(j)}$. We also consider the initial state

$$
\begin{aligned}
\left|\psi_{\beta}\right\rangle= & \cos \theta|g g\rangle \\
& +e^{i \phi} \sin \theta|e e\rangle, \quad \theta \in[0,2 \pi], \phi \in[-\pi, \pi],
\end{aligned}
$$

with the Bell states $\left|\Phi^{+}\right\rangle=\frac{1}{\sqrt{2}}(|g g\rangle+|e e\rangle)$ and $\left|\Phi^{-}\right\rangle=$ $\frac{1}{\sqrt{2}}(|g g\rangle-|e e\rangle)$. As shown in Fig. 2(d), the maximum transported fidelity is independent of $\phi$. The transported fidelity $\mathcal{F}_{\max } \approx 0.954$ at $(\theta=\pi / 4, \phi=0)$ and $(\theta=\pi / 4, \phi=\pi)$ corresponds to the Bell states $\left|\Phi^{ \pm}\right\rangle$, respectively. The lowest $\mathcal{F}_{\max } \approx 0.88$ occurs at $\theta=\pi / 2$, which is reasonable since that corresponds to the case of transporting a two-excitation state $|e e\rangle$, and the probability of excitation leakage via dissipation to the right waveguide port increases when transferring higher-excitation states. Overall, we have shown that good transport of entanglement is possible for all the Bell states.

\section{TRANSPORT OF MULTIPARTITE ENTANGLEMENT WITH CHIRAL COUPLINGS}

Here we demonstrate a generalization of the entanglement transport scheme by using $N$ qubits per node. Intuitively, it is clear that states with permutation invariance and low excitations can be transported well using this scheme. In Fig. 3(a) we show the transport of Dicke states denoted by $\left|{ }^{N} D_{k}\right\rangle$, which is an equal superposition of $k$ excitations over $N$ qubits. It can be seen that the three-qubit $W$ state $\left|W_{3}\right\rangle=$ $\left|{ }^{3} D_{1}\right\rangle$ is transported with a fidelity of $\mathcal{F}_{\max } \approx 0.954$, while the two-excitation states $\left|{ }^{3} D_{2}\right\rangle$ and $\left|{ }^{4} D_{2}\right\rangle$ are transported with a lower fidelity of 0.905 due to increased leakage of excitation from the second node of the quantum network. The $W$ states are extremely useful for quantum information and communication applications as they are more robust states for encoding single-qubit states. Moreover, $W$ states have the unique property (contrary to, say, Greenberger-Horne-Zeilinger states) that even if one particle is lost, the rest of the $N-1$ qubits will remain in the entangled state. In Fig. 3(b) the optimal $g_{j}$ for the transport of $\left|W_{N}\right\rangle$ is plotted. We numerically show that the optimal transport condition for any $\left|W_{N}\right\rangle$ is given by $\sqrt{N} g_{\text {opt }} / \gamma_{R} \approx 0.43$. In fact, any $\left|W_{M}\right\rangle$ can be mapped onto any $\left|W_{N}\right\rangle(M \neq N$ in general) with the same fidelity of 0.954 as long as this condition is satisfied on each node. To study the effects of detuning on $W$-state transport, we add a random fluctuation $\Delta_{a} \in\left[-\delta_{a}, \delta_{a}\right]$ to the qubit frequencies in Fig. 3(c) and $\Delta_{c} \in\left[-\delta_{c}, \delta_{c}\right]$ to the cavity frequencies in Fig. 3(d). The result shows that while the scheme is more robust against fluctuations in cavity frequencies, good transport can also be achieved for $\delta_{a} \leqslant 0.1 \gamma_{R}$.
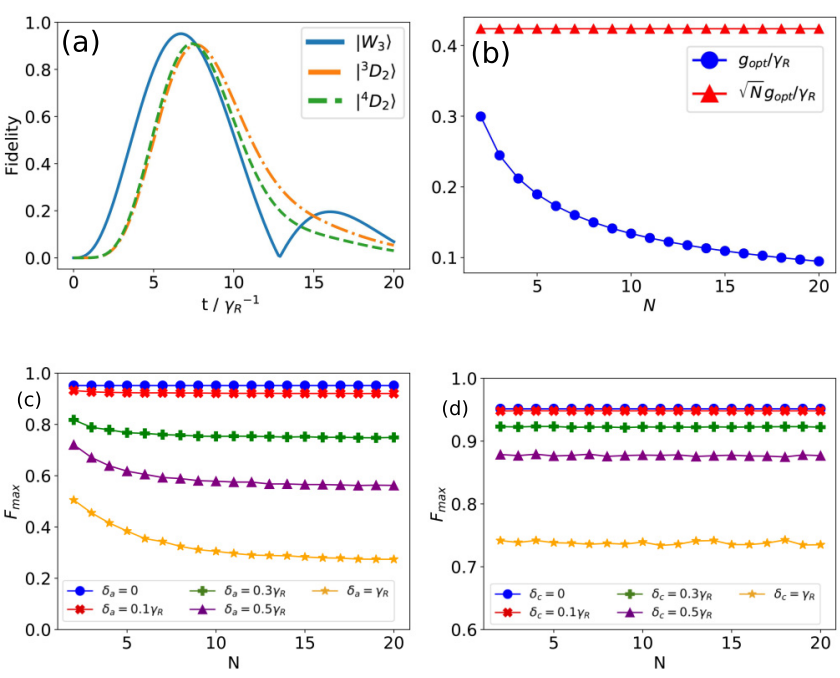

FIG. 3. (a) Transport of Dicke states $\left|W_{3}\right\rangle\left(g_{1}=g_{2}=0.248 \gamma_{R}\right)$, $\left|{ }^{3} D_{2}\right\rangle\left(g_{1}=g_{2}=0.248 \gamma_{R}\right)$, and $\left|{ }^{4} D_{2}\right\rangle\left(g_{1}=g_{2}=0.215 \gamma_{R}\right)$. (b) Optimal $g_{1}=g_{2}$ for $\left|W_{N}\right\rangle$. (c) Effect of random fluctuations in qubit frequencies on fidelity. (d) Effect of random fluctuations in cavity frequencies on fidelity. The other parameters are $\gamma_{L}=0$ and $\Gamma_{j l}=0$.

\section{ROLE OF IMPERFECTIONS}

The analysis in the previous sections neglected qubit losses by assuming that the decay rate of the cavity is much larger than that of the qubit decay rates. Here we look at the entanglement transport with qubit losses. Specifically, we prepare the initial state of $1 a, 2 a$ in the Bell state $\left|\Psi^{+}\right\rangle$and set all qubit decay rates to be equal $\left(\Gamma_{j l}=\Gamma\right)$ for simplicity. The optimal case (from optimization over system parameters) is presented for each value of $\Gamma$. In general, for larger $\Gamma$, the probability of spontaneous emission of the initially excited qubits in the first node increases. Thus, in order to transport the excitation effectively, the excitation should be transferred to the ring cavity before significant qubit decay occurs, resulting in larger optimal coupling $g_{\text {opt }}$.

Increasing the qubit decay rate, the fidelity decreases as shown in Fig. 4(b). Here we set the internodal distance to be at the optimal point $k D=\pi$. A comparison between the chiral $(\chi=1)$ and nonchiral $(\chi=0)$ cases shows that as long as the qubit decay rate is within $\Gamma<0.1 \gamma_{R}$, the chiral system remains advantageous over the ideal nonchiral case
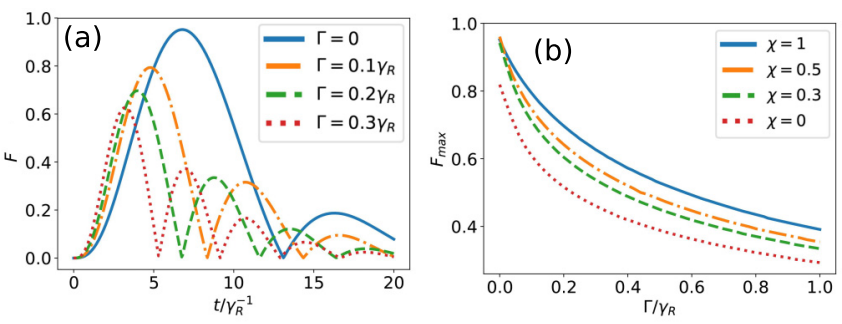

FIG. 4. Effects of qubit losses on entanglement transport of $\left|\Psi^{+}\right\rangle$. (a) Fidelity of $2 a, 2 b$ with chiral coupling. (b) Maximum fidelity of $2 a, 2 b$ against qubit decay rate. The optimized case is shown for each value of $\Gamma$. 
in terms of entanglement transport. As mentioned earlier, the entanglement transport at $k D=\pi$ is also relatively insensitive to small imperfections in chirality. Thus, perfect chirality is not required for the transport scheme to work well.

\section{PROPOSAL FOR EXPERIMENTAL IMPLEMENTATIONS}

Our scheme can be experimentally realized in two different platforms. First, the authors in Ref. [39] recently established a photonic interface of chiral cavity QED using the coupled photonic crystal (PC) and plasmon nanoparticle structure. In this system, the rate of circularly polarized photons emitting into the PC waveguide is one order of magnitude larger than that without the nanoparticle, with about $95 \%$ of photons propagating unidirectionally along the waveguide. In particular, a silver nanoparticle which serves as a nanocavity is embedded inside the W1 PC where the electric field is mainly located with an antisymmetric local helicity distribution. The efficient chiral coupling is controlled by the relative position between the emitter and silver nanoparticle. By applying a strong magnetic field and suitably choosing the laser frequency, two circularly polarized states of the atoms or quantum dots can be generated to emit only clockwise or counterclockwise photons [30].

Another possibility to realize our proposal is by coupling a single ${ }^{85} \mathrm{Rb}$ atom with a microbottle resonator, which is interfaced with a silica nanophotonic tapered fiber. As experimentally demonstrated in Refs. [40,41], chirality naturally arises in such a system due to the strong transverse confinement of the light in the microbottle resonator, which results in different circular polarizations for the clockwise and counterclockwise TM modes in the whispering-gallery mode microresonator. The selection rules for dipole transitions prevent the atom from emitting light into the counterpropagating TM mode. Consequently, the chirality arises because the atom has different interaction cross sections (up to one order of magnitude difference) for the two orthogonal circular polarizations. For our proposal, it suffices to initialize the atoms in the second node in the outermost $m_{F}=3$ Zeeman sublevel of the $F=3$ hyperfine ground state. The excited state corresponds to the $F^{\prime}=4, m_{F}=4$ state. Similarly, we can prepare the ensemble of atoms in the first node in the entangled state. A magnetic field of $B=4.5 \mathrm{G}$ can be applied along the resonator axis to lift the degeneracy.

\section{CONCLUSION}

In this paper we have proposed a protocol for transporting entanglement between the two nodes in an open quantum network, where we demonstrated that dissipation can be useful to achieve the task, contrary to the common notion that dissipation creates decoherence. By coupling ring cavities with a chiral $1 \mathrm{D}$ waveguide, we demonstrated entanglement transport, with the entangled state stored in the atomic ensembles which are coupled to the ring cavities. Consequently, our scheme can be implemented experimentally, by coupling a photonic waveguide with ring cavities which are then coupled to an atomic ensemble, to realize the elementary unit of a quantum node. The communication channel is realized by the chiral waveguide. We have found optimal system parameters for the transport of maximally entangled Bell states and for up to 20-qubit $W$ states. As an application of our results, the quantum transport of $W$ states and Bell states can be exploited to achieve QST of unknown qubit and qutrit states, respectively. We highlight that our proposal requires minimal control over the system parameters, contrary to other proposals which require external pulses with demanding temporal shapes and time-dependent cavity couplings [7-16]. Moreover, since the entanglement transport is achieved dynamically, it is faster compared to its steady-state counterparts, which requires timescales on the order of $10^{2} \gamma_{R}^{-1}[31,42,43]$. Finally, our protocol can easily be applied to long-distance transport by utilizing the Markovianity in cascaded systems. This can potentially be significant for the efficient distribution of entanglement within a quantum network.

\section{ACKNOWLEDGMENTS}

D.A. and W.-K.M. would like to acknowledge MarcAntoine Lemonde for helpful discussions and feedback. The authors thank Jingu Pang for the waveguide diagram. W.-K.M., J.-B.Y., W.Z., and C.E.P. would like to acknowledge support from the National Research Foundation Singapore (Grants No. NRF2017NRFNSFC002-015, No. NRF2016NRF-ANR002, and No. NRF-CRP 14-2014-04) and A*STAR SERC (Grant No. A1685b0005). D.A., L.-C.K., and J.-B.Y. acknowledge support from National Research Foundation and the Ministry of Education, Singapore.

\section{APPENDIX: DERIVATION OF THE EFFECTIVE MASTER EQUATION}

In this Appendix we derive the effective master equation from tracing out the degrees of freedom of the common bath, which in this case is the $1 \mathrm{D}$ waveguide. Note that this derivation is similar to the approach taken in [9]. For simplicity, we neglect qubit decays here and assume that the ring resonators are at a common frequency $\omega_{c}$. The Hamiltonian is given by $H=H_{B}+H_{S}+H_{S B}$, where

$$
\begin{aligned}
H_{S} & =\sum_{j=1}^{2} \sum_{l=1}^{N}\left[\omega_{l}^{(j)} \sigma_{l}^{(j)^{\dagger}} \sigma_{l}^{(j)}+\omega_{c j} a_{j}^{\dagger} a_{j}+g_{l}^{(j)}\left(a_{j}^{\dagger} \sigma_{l}^{(j)}+\text { H.c. }\right)\right], \\
H_{B} & =\sum_{\lambda=L, R} \int d \omega \omega b_{\lambda}^{\dagger}(\omega) b_{\lambda}(\omega)+\sum_{j=1}^{2} \sum_{l=1}^{N} \int d \omega \omega c_{l}^{(j) \dagger}(\omega) c_{l}^{(j)}(\omega), \\
H_{S B} & =i \sum_{j=1}^{2} \sum_{\lambda=L, R} \int d \omega \sqrt{\frac{\gamma_{\lambda}}{2 \pi}}\left[b_{\lambda}^{\dagger}(\omega) e^{-i k x_{j}} a_{j}-\text { H.c. }\right]+i \sum_{j=1}^{2} \sum_{l=1}^{N} \int d \omega \sqrt{\frac{\Gamma_{j l}}{2 \pi}}\left[c_{l}^{(j) \dagger}(\omega) \sigma_{l}^{(j)}-\text { H.c. }\right] .
\end{aligned}
$$


Setting $\Gamma_{j l}=0$ from the Hamiltonian in Eq. (A1), choosing a frame rotating with the cavity and bath, i.e., $U=$ $\exp \left\{i\left[\sum_{j} \omega_{c j} a_{j}^{\dagger} a_{j}+\sum_{\lambda} \int d \omega \omega b_{\lambda}^{\dagger}(\omega) b_{\lambda}(\omega)\right]\right\}$, and applying the transformation $H=U H U^{\dagger}-i \dot{U} U^{\dagger}$, we have

$$
\tilde{H}_{S B}(t)=i \sum_{\lambda, j} \int d \omega \sqrt{\frac{\gamma_{\lambda}}{2 \pi}}\left[b_{\lambda}^{\dagger}(\omega) a_{j} e^{i\left(\omega-\omega_{c}\right) t} e^{-i \omega x_{j} / v}-e^{i\left(\omega-\omega_{c}\right) t} e^{i \omega x_{j} / v} a_{j}^{\dagger} b_{\lambda}(\omega)\right] .
$$

From the Heisenberg equations of motion we have

$$
\dot{b}_{\lambda}(\omega, t)=i\left[H, b_{\lambda}(\omega, t)\right]=\sum_{j=1,2} \sqrt{\frac{\gamma_{\lambda}}{2 \pi}} a_{j}(t) e^{i\left(\omega-\omega_{c}\right) t} e^{-i \omega x_{j} / v},
$$

which can be formally integrated to obtain

$$
b_{\lambda}(\omega, t)=b_{\lambda}(\omega, 0)+\int_{0}^{t} d s \sum_{j} \sqrt{\frac{\gamma_{\lambda}}{2 \pi}} a_{j}(s) e^{i\left(\omega-\omega_{c}\right) s} e^{-i \omega x_{j} / v} .
$$

For an arbitrary system operator $X(t)$, the Heisenberg equation reads

$$
\dot{X}(t)=\sum_{\lambda, j} \int d \omega \sqrt{\frac{\gamma_{\lambda}}{2 \pi}}\left\{b_{\lambda}^{\dagger}(\omega, t) e^{i\left(\omega-\omega_{c}\right) t} e^{-i \omega x_{j} / v}\left[X(t), a_{j}(t)\right]-b_{\lambda}(\omega, t) e^{-i\left(\omega-\omega_{c}\right) t} e^{i \omega x_{j} / v}\left[X(t), a_{j}^{\dagger}(t)\right]\right\} .
$$

Substituting $b_{\lambda}(\omega, t)$ into $\dot{X}(t)$ and defining $b_{\lambda}(t) \equiv \frac{1}{\sqrt{2 \pi}} \int d \omega b_{\lambda}(\omega) e^{-i\left(\omega-\omega_{j}\right) t}$ and $k=\omega_{0} / v$, we have

$$
\begin{aligned}
\dot{X}(t)= & \sum_{\lambda, j} \sum_{\lambda, j} \sqrt{\gamma_{\lambda}} b_{\lambda}^{\dagger}\left(t-x_{j} / v\right) e^{-i k x_{j}}\left[X(t), a_{j}(t)\right]-\left[X(t), a_{j}^{\dagger}(t)\right] b_{\lambda}\left(t-x_{j} / v\right) e^{i k x_{j}} \\
& +\sum_{\lambda, j, l} \frac{\gamma_{\lambda}}{2 \pi} \int_{0}^{t} d s \int d \omega e^{i\left(\omega-\omega_{c}\right)(t-s)} e^{-i \omega x_{j l} / v} a_{l}^{\dagger}(s)\left[X(t), a_{j}(t)\right]-e^{-i\left(\omega-\omega_{c}\right)(t-s)} e^{i \omega x_{j l} / v} a_{l}(s)\left[X(t), a_{j}^{\dagger}(t)\right] .
\end{aligned}
$$

We can perform the Born-Markov approximation by treating the time delay $x_{j l} / v$ between the two atoms to be very small. Thus,

$$
\sum_{l} \frac{1}{2 \pi} \int_{0}^{t} d s \int d \omega e^{i\left(\omega-\omega_{j}\right)(t-s)} e^{-i \omega x_{j l} / v} a_{l}^{\dagger}(s)=\sum_{l} \int_{0}^{t} d s \delta\left(t-x_{j l} / v-s\right) e^{-i k x_{j l}} a_{l}^{\dagger}(s) \approx \frac{1}{2} a_{l}^{\dagger}(t)+\sum_{l} \theta\left(x_{j l} / v\right) e^{-i k x_{j l}} a_{l}^{\dagger}(t),
$$

where the first term is the contribution from $x_{j l} / v<0$ and the second term is from $x_{j l} / v>0$. The Markov approximation is also applied to the second term $\sigma_{l}^{\dagger}\left(t-x_{j l} / v\right) \rightarrow \sigma_{l}^{\dagger}(t)$. Next we substitute this into the equation for $\dot{X}(t)$ and take averages. Since the bath is initially in the vacuum, $\left\langle b_{\lambda}(t)\right\rangle=0$. Thus,

$$
\langle\dot{X}(t)\rangle=\sum_{\lambda j} \frac{\gamma_{\lambda}}{2}\left\{\left\langle a_{j}^{\dagger}(t)\left[X(t), a_{j}(t)\right]\right\rangle-\left\langle\left[X(t), a_{j}^{\dagger}(t)\right] a_{j}(t)\right\rangle\right\}+\sum_{\lambda j l, x_{j}>x_{l}} \gamma_{\lambda}\left\{e^{-i k x_{j l}}\left\langle a_{l}^{\dagger}(t)\left[X(t), a_{j}(t)\right]\right\rangle-e^{i k x_{j l}}\left\langle\left[X(t), a_{j}^{\dagger}(t)\right] a_{l}(t)\right\rangle\right\} .
$$

To obtain the master equation, we first note that the average is the same in both the Schrödinger picture and the Heisenberg picture, and thus $\operatorname{Tr}[X(t) \rho(0)]=\operatorname{Tr}[X \rho(t)]$, that is, we can move the time dependence from the system operator to the density operator. For example, the first term on the right-hand side can be written as

$$
\left\langle a_{j}^{\dagger}(t)\left[X(t), a_{j}(t)\right]\right\rangle=\operatorname{Tr}\left[a_{j}^{\dagger} X a_{j} \rho(t)-a_{j} X a_{j}^{\dagger} \rho(t)\right]=\operatorname{Tr}\left\{X\left[a_{j}, \rho(t) a_{j}^{\dagger}\right]\right\}
$$

using the cyclic property of trace. Doing this for all the terms and noting that the equation holds for all $X(t)$, we have

$$
\dot{\rho}(t)=-i\left[H_{S}, \rho(t)\right]+\sum_{\lambda j} \frac{\gamma_{\lambda}}{2}\left\{\left[a_{j}, \rho(t) a_{j}^{\dagger}\right]-\left[a_{j}^{\dagger}, a_{j} \rho(t)\right]\right\}+\sum_{\lambda j l, x_{j}>x_{l}} \gamma_{\lambda}\left\{e^{-i k x_{j l}}\left[\sigma_{j}, \rho(t) a_{l}^{\dagger}\right]-e^{i k x_{j l}}\left[a_{j}^{\dagger}, a_{l} \rho(t)\right]\right\},
$$

where the last term describes the effective long-range interactions between the two resonators mediated by the waveguide. By separating the interaction term into coherent and incoherent parts, the effective master equation described in the main text is obtained.

[1] H. J. Kimble, Nature (London) 453, 1023 (2008).

[2] A. Reiserer and G. Rempe, Rev. Mod. Phys 87, 1379 (2015).
[3] M. A. Nielsen and I. L. Chuang, Quantum Computation and Quantum Information (Cambridge University Press, Cambridge, 2002). 
[4] H.-P. Breuer and F. Petruccione, The Theory of Open Quantum Systems (Oxford University Press, Oxford, 2002).

[5] C. Gonzalez-Ballestero, F. J. García-Vidal, and E. Moreno, New J. Phys. 15, 073015 (2013).

[6] Y.-L. L. Fang, F. Ciccarello, and H. U. Baranger, New J. Phys. 20, 043035 (2018).

[7] J. I. Cirac, P. Zoller, H. J. Kimble, and H. Mabuchi, Phys. Rev. Lett. 78, 3221 (1997).

[8] G. M. Nikolopoulos and I. E. Jex, Quantum State Transfer and Network Engineering (Springer, Berlin, 2014).

[9] B. Vermersch, P.-O. Guimond, H. Pichler, and P. Zoller, Phys. Rev. Lett. 118, 133601 (2017).

[10] C. Dlaska, B. Vermersch, and P. Zoller, Quantum Sci. Technol. 2, 015001 (2017).

[11] K. Stannigel, P. Rabl, A. S. Sørensen, P. Zoller, and M. D. Lukin, Phys. Rev. Lett. 105, 220501 (2010).

[12] K. Stannigel, P. Rabl, A. S. Sørensen, M. D. Lukin, and P. Zoller, Phys. Rev. A 84, 042341 (2011).

[13] N. Y. Yao, C. R. Laumann, A. V. Gorshkov, H. Weimer, L. Jiang, J. I. Cirac, P. Zoller, and M. D. Lukin, Nat. Commun. 4, 1585 (2013).

[14] T. Ramos, B. Vermersch, P. Hauke, H. Pichler, and P. Zoller, Phys. Rev. A 93, 062104 (2016).

[15] H. Zheng and H. U. Baranger, Phys. Rev. Lett. 110, 113601 (2013).

[16] T. van Leent, M. Bock, R. Garthoff, K. Redeker, W. Zhang, T. Bauer, W. Rosenfeld, C. Becher, and H. Weinfurter, Phys. Rev. Lett. 124, 010510 (2020).

[17] Z.-L. Xiang, M. Zhang, L. Jiang, and P. Rabl, Phys. Rev. X 7, 011035 (2017).

[18] J. Hofmann, M. Krug, N. Ortegel, L. Gérard, M. Weber, W. Rosenfeld, and H. Weinfurter, Science 337, 72 (2012).

[19] S. Ritter, C. Nölleke, C. Hahn, A. Reiserer, A. Neuzner, M. Uphoff, M. Mücke, E. Figueroa, J. Bochmann, and G. Rempe, Nature (London) 484, 195 (2012).

[20] W. Rosenfeld, D. Burchardt, R. Garthoff, K. Redeker, N. Ortegel, M. Rau, and H. Weinfurter, Phys. Rev. Lett. 119, 010402 (2017).

[21] S. Bose, Contemp. Phys. 48, 13 (2007).
[22] M. Rafiee, M. Soltani, H. Mohammadi, and H. Mokhtari, Eur. Phys. J. D 63, 473 (2011).

[23] A. Bayat and S. Bose, Adv. Math. Phys. 2010, 127182 (2010).

[24] R. Sousa and Y. Omar, New J. Phys. 16, 123003 (2014).

[25] Y.-H. Ji and Y.-M. Liu, Optik 126, 2414 (2015).

[26] Z.-X. Man, N. B. An, Y.-J. Xia, and J. Kim, Phys. Lett. A 378, 2063 (2014).

[27] R. Vieira and G. Rigolin, Phys. Lett. A 382, 2586 (2018).

[28] L. Banchi, T. J. G. Apollaro, A. Cuccoli, R. Vaia, and P. Verrucchi, New J. Phys. 13, 123006 (2011).

[29] P. Lodahl, S. Mahmoodian, S. Stobbe, A. Rauschenbeutel, P. Schneeweiss, J. Volz, H. Pichler, and P. Zoller, Nature (London) 541, 473 (2017).

[30] I. Söllner, S. Mahmoodian, S. L. Hansen, L. Midolo, A. Javadi, G. Kiršanskè, T. Pregnolato, H. El-Ella, E. H. Lee, J. D. Song, S. Stobbe, and P. Lodahl, Nat. Nanotechnol. 10, 775 (2015).

[31] H. Pichler, T. Ramos, A. J. Daley, and P. Zoller, Phys. Rev. A 91, 042116 (2015).

[32] S. Mahmoodian, P. Lodahl, and A. S. Sørensen, Phys. Rev. Lett. 117, 240501 (2016).

[33] C. Gonzalez-Ballestero, A. Gonzalez-Tudela, F. J. GarciaVidal, and E. Moreno, Phys. Rev. B 92, 155304 (2015).

[34] H. J. Carmichael, Phys. Rev. Lett. 70, 2273 (1993).

[35] C. W. Gardiner, Phys. Rev. Lett. 70, 2269 (1993).

[36] H. J. Carmichael, Statistical Methods in Quantum Optics 2: NonClassical Fields (Springer, Berlin, 2007).

[37] M. Arcari, I. Söllner, A. Javadi, S. Lindskov Hansen, S. Mahmoodian, J. Liu, H. Thyrrestrup, E. H. Lee, J. D. Song, S. Stobbe, and P. Lodahl, Phys. Rev. Lett. 113, 093603 (2014).

[38] W. K. Wootters, Phys. Rev. Lett. 80, 2245 (1998).

[39] F. Zhang, J. Ren, L. Shan, X. Duan, Y. Li, T. Zhang, Q. Gong, and Y. Gu, Phys. Rev. A 100, 053841 (2019).

[40] C. Sayrin, C. Junge, R. Mitsch, B. Albrecht, D. O'Shea, P. Schneeweiss, J. Volz, and A. Rauschenbeutel, Phys. Rev. X 5, 041036 (2015).

[41] C. Junge, D. O'Shea, J. Volz, and A. Rauschenbeutel, Phys. Rev. Lett. 110, 213604 (2013).

[42] Y. Matsuzaki, V. M. Bastidas, Y. Takeuchi, W. J. Munro, and S. Saito, arXiv:1810.02995.

[43] D. X. Li and X. Q. Shao, Phys. Rev. A 99, 032348 (2019). 